\title{
PREVALENCE AND RECOVERABILITY OF SYNTACTIC PARAMETERS IN SPARSE DISTRIBUTED MEMORIES
}

\author{
JEONG JOON PARK, RONNEL BOETTCHER, ANDREW ZHAO, ALEX MUN, KEVIN YUH, \\ VIBHOR KUMAR, MATILDE MARCOLLI
}

\begin{abstract}
We propose a new method, based on Sparse Distributed Memory (Kanerva Networks), for studying dependency relations between different syntactic parameters in the Principles and Parameters model of Syntax. We store data of syntactic parameters of world languages in a Kanerva Network and we check the recoverability of corrupted parameter data from the network. We find that different syntactic parameters have different degrees of recoverability. We identify two different effects: an overall underlying relation between the prevalence of parameters across languages and their degree of recoverability, and a finer effect that makes some parameters more easily recoverable beyond what their prevalence would indicate. We interpret a higher recoverability for a syntactic parameter as an indication of the existence of a dependency relation, through which the given parameter can be determined using the remaining uncorrupted data.
\end{abstract}

\section{INTRODUCTION}

1.1. Syntactic Parameters of World Languages. The general idea behind the Principles and Parameters approach to Syntax, 2], [3], is the encoding of syntactic properties of natural languages as a string of binary variables, the syntactic parameters. This model is sometimes regarded as controversial, and some schools of Linguistics have, consequently, moved towards other possible ways of modeling syntax. However, syntactic parameters remain more suitable than other concurrent models from the point of view of a mathematical approach, as we set out to demonstrate in a series of related papers [19], [22], [26]. Among the shortcomings ascribed to the Principles and Parameters model (see for instance [10]) is the fact that it has not been possible, so far, to identify a complete set of such syntactic parameters, even though extensive lists of parameters are classified and recorded for a large number of natural languages. It is also unclear what relations exist between parameters and whether there is a natural choice of a set of independent variables among them.

At present, sufficiently rich databases of syntactic parameters of world languages are available, most notably the "Syntactic Structures of the World's Languages" (SSWL) database 29] (recently migrated to TerraLing [30]) and the "World Atlas of Language Structures" (WALS) [9]. This makes it possible to reconsider the problem of syntactic parameters, loosely formulated as understanding the geometry of the parameter space and how parameters are distributed across language families, with modern methods of data analysis. For example, topological data analysis was applied to syntactic parameters in [22]. In the present paper, the main tool of analysis we will employ to study relations between syntactic parameters will be Kanerva Networks.

In this paper we selected a list of 21 syntactic parameters, mostly having to do with word order relations (see $\$ 2.1$ below of a detailed discussion of the chosen parameters), and a list of 166 languages, for which the values of these parameters are recorded in the SSWL database (the languages used are listed in the Appendix). The parameters are selected so that they clearly are not an independent set of binary variables (see the discussion in $\$ 2.2$ below). The languages are selected so that they cut across a broad range of different linguistic families. By storing the 
data of syntactic parameters for this group of languages in a Kanerva Network, we can test for recoverability when one of the binary variables is corrupted. We find an overall relation between recoverability and prevalence across languages, which depends on the functioning of the sparse distributed memory. Moreover, we also see a further effect, which deviates from a simple relation with the overall prevalence of a parameter. This shows that certain syntactic parameters have a higher degree of recoverability in a Kanerva Network. This property can be interpreted as a consequence of existing underlying dependence relations between different parameters. With this interpretation, one can envision a broader use of Kanerva Networks as a method to identify further, and less clearly visible, dependence relations between other groups of syntactic parameters.

Another reason why it is interesting to analyze syntactic parameters using Kanerva Networks is the widespread use of the latter as models of human memory, [7, [13, [15. In view of the problem of understanding mechanism of language acquisition, and how the syntactic structure of language may be stored in the human brain, sparse distributed memories appear to be a promising candidate for the construction of effective computational models.

Acknowledgment. This work was performed as part of the activities of the last author's Mathematical and Computational Linguistics lab and CS101/Ma191 class at Caltech. The last author is partially supported by NSF grants DMS-1201512 and PHY-1205440.

\section{Syntactic Parameters}

2.1. Choice of parameters. For the purpose of this study, we focused on a list of 21 syntactic parameters, which are listed in the SSWL database as

01 Subject-Verb

02 Verb-Subject

03 Verb-Object

04 Object-Verb

05 Subject-Verb-Object

06 Subject-Object-Verb

07 Verb-Subject-Object

08 Verb-Object-Subject

09 Object-Subject-Verb

10 Object-Verb-Subject

11 Adposition-Noun-Phrase

12 Noun-Phrase-Adposition

13 Adjective-Noun

14 Noun-Adjective

15 Numeral-Noun

16 Noun-Numeral

17 Demonstrative-Noun

18 Noun-Demonstrative

19 Possessor-Noun

20 Noun-Possessor

A01 Attributive-Adjective-Agreement

The first 10 parameters on this list deal with word order properties. Subject-Verb has the value 1 when in a clause with an intransitive verb the order subject followed by verb can be used in a neutral context, and value 0 otherwise. Verb-Subject has value 1 when, in the same setting, the 
order verb followed by subject can be used. For example: English has value 1 for Subject-Verb and value 0 for Verb-Subject while Italian has value 1 for both parameters. Verb-Object has value 1 when a main verb (not the auxiliary) can precede its object in a neutral context, and 0 otherwises; while Object-Verb has value 1 if the main verb can follow its object in a neutral context, and 0 otherwise. English has Verb-Object value 1 and Object-Verb value 0; German has value 1 for both; Japanese has Verb-Object set to 0 and Object-Verb value 1. The remaining 6 parameters in this group describe the different word order structures SVO, SOV, VSO, VOS, OSV, OVS: each of these parameters has value 1 when the corresponding word order can be used in a neutral context, and value 0 otherwise. These word order parameters have very different distribution among the world languages: of the six possible word orders listed above, it is estimated that around $45 \%$ of the world languages follow the SOV order, $42 \%$ the SVO, $9 \%$ have VSO, 3\% have VOS, only $1 \%$ follow the OVS order, and the remaining possibility, OSV, is extremely rare, estimated at only $0.2 \%$, see [28]. We will return to discuss how the relative frequencies of different parameters, within the group of languages that we consider in this paper, affect the behavior in the Kanerva Network. The frequencies of the 21 parameters within the group of languages used for this study (see the list in the Appendix) are reported in the table below.

\begin{tabular}{|c|c|}
\hline Parameter & Frequency \\
\hline \hline$[01]$ Subject-Verb & 0.64957267 \\
\hline$[02]$ Verb-Subject & 0.31623933 \\
\hline$[03]$ Verb-Object & 0.61538464 \\
\hline$[04]$ Object-Verb & 0.32478634 \\
\hline$[05]$ Subject-Verb-Object & 0.56837606 \\
\hline$[06]$ Subject-Object-Verb & 0.30769232 \\
\hline$[07]$ Verb-Subject-Object & 0.1923077 \\
\hline$[08]$ Verb-Object-Subject & 0.15811966 \\
\hline$[09]$ Object-Subject-Verb & 0.12393162 \\
\hline$[10]$ Object-Verb-Subject & 0.10683761 \\
\hline$[11]$ Adposition-Noun-Phrase & 0.58974361 \\
\hline$[12]$ Noun-Phrase-Adposition & 0.2905983 \\
\hline$[13]$ Adjective-Noun & 0.41025642 \\
\hline$[14]$ Noun-Adjective & 0.52564102 \\
\hline$[15]$ Numeral-Noun & 0.48290598 \\
\hline$[16]$ Noun-Numeral & 0.38034189 \\
\hline$[17]$ Demonstrative-Noun & 0.47435898 \\
\hline$[18]$ Noun-Demonstrative & 0.38461539 \\
\hline$[19]$ Possessor-Noun & 0.38034189 \\
\hline$[20]$ Noun-Possessor & 0.49145299 \\
\hline$[$ A 01] Attributive-Adjective-Agreement & 0.46581197 \\
\hline
\end{tabular}

The Adposition-Noun-Phrase parameter is set to 1 in a language, when there are adpositions that precede the noun phrase they occurs with, while the Noun-Phrase-Adposition parameter is set to 1 when there are adpositions that follow the noun phrase. Both Adposition-Noun-Phrase and Noun-Phrase-Adposition can have value 1 in a language that has both prepositions and postpositions. The pair of parameters Adjective-Noun and Noun-Adjective regulate whether an adjective can precede (respectively, follow) the noun it modifies in a neutral context. Similarly, NumeralNoun and Noun-Numeral are set to 1 when there are, in the language, cardinal numerals that precede (respectively, follow) the noun they modify in a neutral context. The same for the pairs 
Demonstrative-Noun and Noun-Demonstrative, and Possessor-Noun and Noun-Possessor with respect to demonstratives (respectively, possessors) and the noun they modify. Finally, the parameter Attributive-Adjective-Agreement is set to 1 for a language when there are attributive adjectives that show agreement with (some of) the nouns they modify. For example, this parameter is 0 for English and 1 for Italian.

A complete list of the syntactic parameters recorded in the SSWL database and their linguistic meaning is available at http://sswl.railsplayground.net/browse/properties and in TerraLing http://www.terraling.com/groups/9/properties

This particular choice of languages from the SSWL database is motivated by the fact that, for this list, there is a complete mapping of the values of the 21 syntactic parameters listed above. This makes it possible to construct a Kanerva network with enough data points in it to carry out our intended analysis.

2.2. Parameters and Dependencies. There is clearly some degree of dependence between the 6 word order parameters SVO, SOV, VSO, VOS, OSV, OVS and the previous 4 parameters in the list, so that these cannot be all completely independent binary variables. However, this dependence relation is more subtle than it might appear at first. To illustrate the point with an example, consider the case of the languages English and Italian. Both have 1 for SVO and 0 for VSO, but as mentioned above English has value 1 for Subject-Verb and value 0 for Verb-Subject, while Italian has value 1 for both parameters. This means that the relation between these parameters is not simply a fixed algebraic dependence relation (unlike the entailment of parameters that we analyzed in [26], for example). Rather, there may be relations that are expressible probabilistically, in terms of frequencies and correlations. This is the type of relations that we seek to identify with the use of sparse distributed memories.

Our purpose in this study is to determine how much the presence of dependencies between the syntactic parameters is detectable through a Kanerva Network model, by measuring recoverability of some parameters in terms of the remaining ones.

\section{Sparse Distributed Memory}

Kanerva Networks (or Sparse Distributed Memory) were developed by Pentti Kanerva in 1988, [12], 13], as a mathematical model of human long term memory. The model allows for approximate accuracy storage and recall of data at any point in a high dimensional space, using fixed hard locations distributed randomly throughout the space. During storage of a datum, hard locations "close" to the datum encode information about the data point. Retrieval of information at a location in the space is performed by pooling nearby hard locations and aggregating their encoded data. The mechanism allows for memory addressability of a large memory space with reasonable accuracy in a sparse representation.

Kanerva Networks model human memory in the following way: a human thought, perception, or experience is represented as an (input) feature vector - a point in a high dimensional space. Concepts stored by the brain are also represented as feature vectors, and are usually stored relatively far from each other in the high dimensional space (the mind). Thus, addressing the location represented by the input vector will yield, to a reasonable degree of accuracy, the concept stored near that location. Thus, Kanerva Networks model the fault tolerance of the human mind - the mind is capable of mapping imprecise input experiences to well defined concepts. For a short introduction to Kanerva Networks aimed at a general public, see $\S 13$ of [6].

More precisely, the functioning of Kanerva Network models can be summarized as follows. Over the field $\mathbb{F}_{2}=\{0,1\}$, consider a vector space (Boolean space) $\mathbb{F}_{2}^{N}$ of sufficiently large dimension $N$. 
Inside $\mathbb{F}_{2}^{N}$, choose a uniform random sample of $2^{k}$ hard locations, with $2^{k}<<2^{N}$. Compute the median Hamming distance between hard locations. The access sphere of a point in the space $\mathbb{F}_{2}^{N}$ is a Hamming sphere of radius slightly larger than this median value (see $\S 6$ of [12] for some precise estimates). When writing to the network at some location $X$ in the space $\mathbb{F}_{2}^{N}$, data is distributively stored by writing to all hard locations within the access sphere of that point $X$. Namely, each hard location stores $N$ counters (initialized to 0 ), and all hard locations within the access sphere of $X$ have their $i$-th counter incremented or decremented by 1 , depending on the value of the $i$-th bit of $X$, see $§ 3.3 .1$ of [13]. When the operation is performed for a set of locations, each hard location stores a datum whose $i$-th entry is determined by the majority rule of the corresponding $i$-th entries for all the stored data. One reads at a location $Y$ in the network a new datum, whose $i$-th entry is determined by comparing 0 to the $i$-th counters of all the hard locations that fall within the access sphere of $Y$, that is, the $i$-th entry read at $Y$ is itself given by the majority rule on the $i$-th entries of all the data stored at all the hard locations accessible from $Y$. For a more detailed account, see [12], [13], and the summary in $\S 13$ of [6].

The network is typically successful in reconstructing stored data, because intersections between access spheres are infrequent and small. Thus, copies of corrupted data in hard locations within the access sphere of a stored datum $X$ are in the minority with respect to hard locations faithful to $X$ 's data. When a datum is corrupted by noise (i.e. flipping bit values randomly), the network is sometimes capable of correctly reconstructing these corrupted bits. The ability to reconstruct certain bits hints that these bits are derived from the remaining, uncorrupted bits in the data.

In addition to modeling human memory in applications to neuroscience and neural computation (see for instance [17]), Kanerva networks have been used in various other contexts, such as weather prediction [25], robotics [21], and as machine-learning tools, in comparison to other forms of associative memory, [4], [11], [15]. Most applications of Kanerva networks in the literature have focused on models of memory and of data storage and recovery. While some applications to Linguistics have been developed, for instance in the setting of speech recognition [24], Kanerva networks have not been previously used to analyze syntactic structures and identify dependencies between syntactic parameters.

3.1. Detecting Parameter Dependencies. Although Kanerva Networks were originally developed for and motivated by human memory, they are also a valuable general tool for detecting dependencies in a high-dimensional data sets. The reasons for this can be found in the literature on Kanerva Networks, see for instance the discussion in [11].

In the present paper, we treat each language, and its corresponding list of syntactic parameters, as a single data point in the network. Concretely, each data point is a concatenated binary string of all the values, for that particular language, of the 21 syntactic parameters listed in $\$ 1.1$.

As we recalled above, a Kanerva network operates by writing to uniformly random hard locations within a Hamming sphere of specified radius centered at the write location (specified by a bitstring), and reading from hard locations within a Hamming sphere centered at the read location, returning the majority rule derived from the data points for each of the individual bits.

Regardless of how well this is representative of human memory, this system can demonstrate a clear correlation (i.e. dependence) between certain parameters. Observe that, if we had written to clusters of data points in the space, interpreted as separate syntactic families of languages, then reading from locations in the vicinity of the locations of these clusters would result in reading back a necessarily correlated set of parameter values, due to the each parameters being determined by the locally smaller set of hard locations. Here, by syntactic families, we do not necessarily mean historical-linguistic families, but rather families of languages whose data set cluster together in the Kanerva Network space. How well such groupings reflect historical-linguistic families remains an 


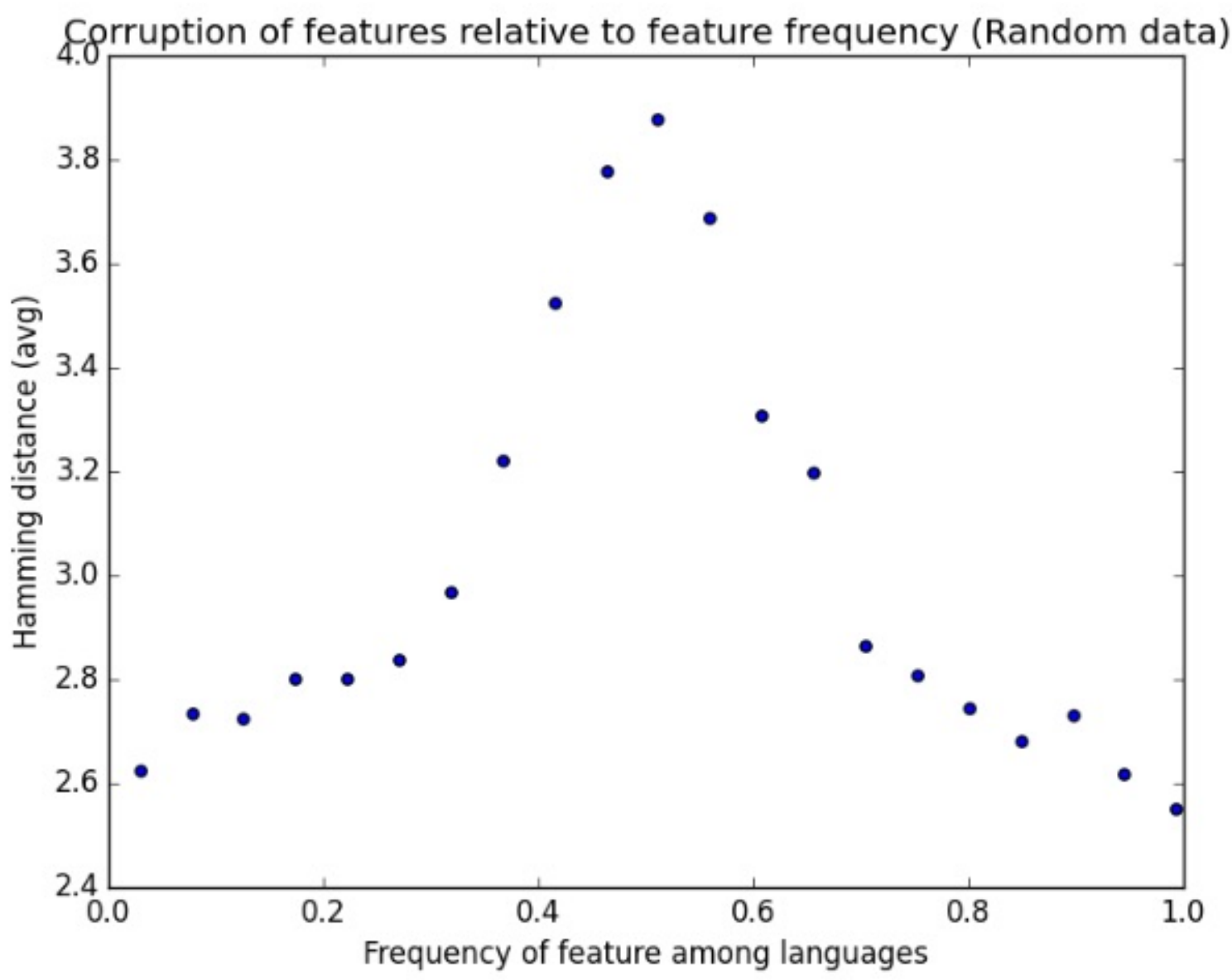

Figure 1. Prevalence and recoverability in a Kanerva Network (random data).

issue for future investigation. If the original location came from a cluster or family of languages, then we would expect to see corrupted bits recovered, indicating that this particular subset of bits is dependent on the rest, i.e. that the parameters are not independent since there exists a non-zero correlation between their values.

\section{Implementation Method}

We considered 166 languages from the SSWL database, which have a complete mapping of the 21 syntactic parameters discussed in 81.1 . These provide 166 data points in a Kanerva Network with Boolean space $\mathbb{F}_{2}^{21}$. The complete list of languages used is reported in the Appendix.

The python/c sdm sparse distributed memory library' was used to simulate the Kanerva network. The current state of the library at the time of the experiment was not functional, so the last working version from January 31, 2014 was used. The library was initialized with an access sphere of $n / 4$, where $n$ is the median hamming distance between items. This was the optimal value we could work with, because larger values resulted in an excessive number of hard locations being in the sphere, which the library was unable to handle.

Three different methods of corruption were tested. First, the correct data was written to the Kanerva network, then reads at corrupted locations were tested. A known language bit-string, with

\footnotetext{
${ }^{1}$ https://github.com/msbrogli/sdm
} 


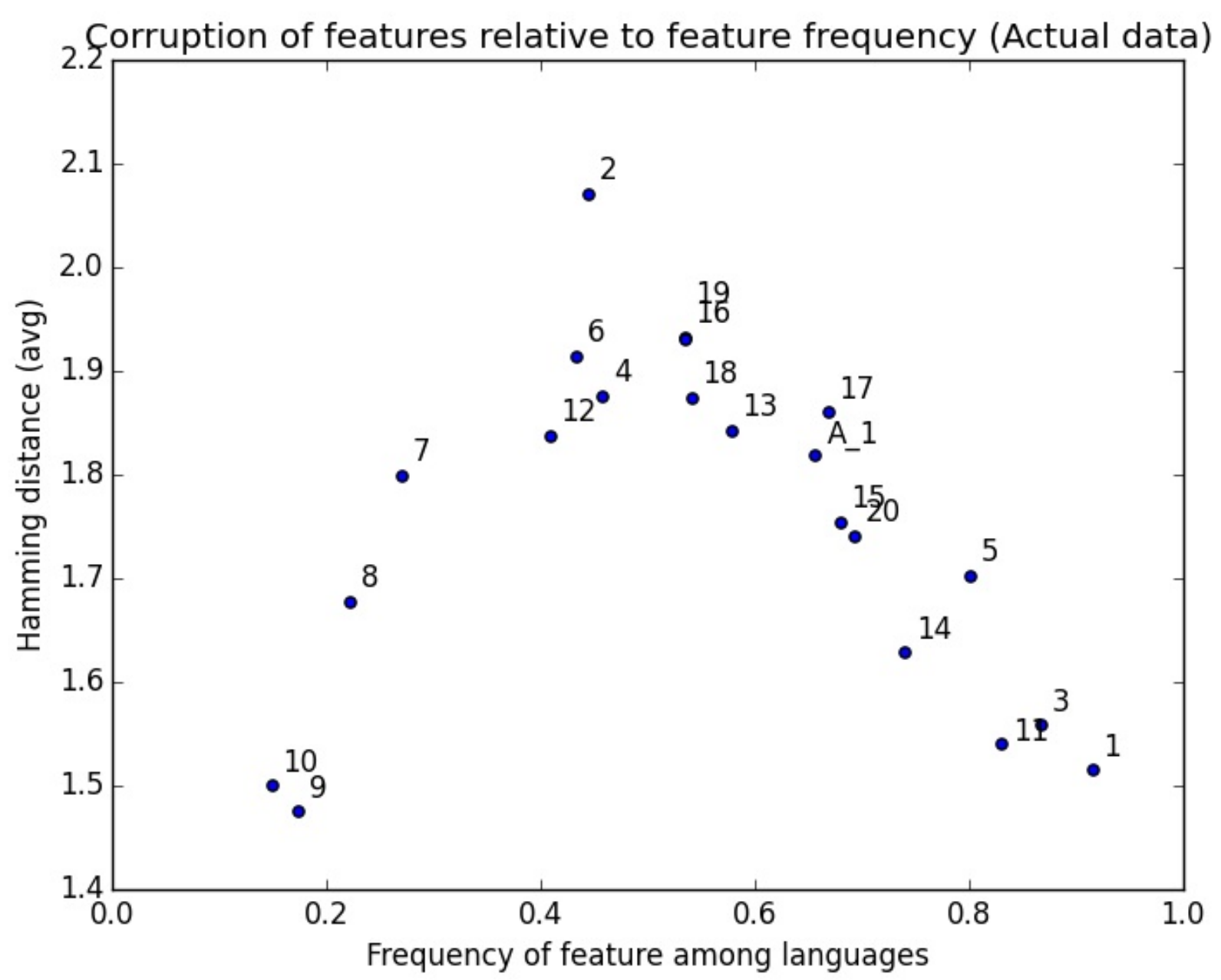

Figure 2. Prevalence and recoverability for syntactic parameters in a Kanerva Network.

a single corrupted bit, was used as the read location, and the result of the read was compared to the original bit-string in order to test bit recovery. The average Hamming distance resulting from the corruption of a given bit, corresponding to a particular syntactic parameter, was calculated across all languages.

In order to test for relationships independent of the prevalence of the features, another test was run that normalized for this. For each feature, a subset of languages of fixed size was chosen randomly such that half of the languages had that feature. Features that had too few languages with or without the feature to reach the chosen fixed size were ignored for this purpose. For this test, a fixed size of 95 languages was chosen, as smaller sizes would yield less significant results, and larger sizes would result in too many languages being skipped. The languages were then written to the Kanerva network and the recoverability of that feature was measured.

Finally, to check whether the different recovery rates we obtained for different syntactic parameters were really a property of the language data, rather than of the Kanerva network itself, the test was run again with random data generated with an approximately similar distribution of bits. In this test, the general relationship of Figure 1 was observed. This indicates that the general shape of the curve may be a property of the Kanerva network. The magnitude of the values for the actual data, however, is very different, see Figure 2. This indicates that the recoverability 
rates observed for the syntactic parameters are begin influenced by the language data, hence they should correspond to actual syntactic properties.

\section{Summary of Main Results}

Summarizing, the main results we obtained in the analysis of the selected data of languages and parameters identifies two different effects on the recoverability of syntactic parameters in Kanerva Networks.

5.1. Large scale structure: prevalence and recoverability. The first effect is a general relation between prevalence of parameters across languages and recoverability in sparse distributed memories. This is a general effect that depends on the functioning of Kanerva Networks and can be seen using random data with the same frequencies as the chosen set of parameters. The curve expressing recoverability as a function of prevalence using random data (Figure 1) indicates the overall underlying effect. This phenomenon seems in itself interesting, given ongoing investigations on how prevalence rates of different syntactic parameters may correlate to neuroscience models, see for instance [16].

5.2. Smaller scale structures of recoverability. In addition to the large scale relationship between prevalence of feature and recoverability mentioned above, the variation of the recoverability values from the general trend is consistent and indicates a second order relationship, which we see in the plot of the real data of syntactic parameters in Figure 2. A far smaller variation from a smooth curve was observed when using random input data as in Figure 1. The normalized test indicates a smaller but still significant variation in feature recoverability even when all features considered had the same prevalence among the dataset.

5.3. Recoverability scores. The resulting levels of recoverability of the syntactic parameters are listed in the table below, and displayed in Figure 3. The results of the normalized test are listed, for a selection of parameters, in the second table and displayed in Figure 4. To each parameter we assign a score, obtained by computing the average Hamming distance between the resulting bit-vector in the corruption experiment and the original one. The lower the score, the more easily recoverable a parameter is from the uncorrupted data, hence from the other parameters. 

[02] VerbSubject
[16] NounNumeral
[19] PossessorNoun
[06] SubjectObjectVerb
[17] DemonstrativeNoun
[18] NounDemonstrative
[04] ObjectVerb
[13] AdjectiveNoun
[12] NounPhraseAdposition
[A01] AttributiveAdjectiveAgreement
[07] VerbSubjectObject
[15] NumeralNoun
[20] NounPossessor
[05] SubjectVerbObject
[08] VerbObjectSubject
[14] NounAdjective
[03] VerbObject
[11] AdpositionNounPhrase
[01] SubjectVerb
[10] ObjectVerbSubject
[09] ObjectSubjectVerb

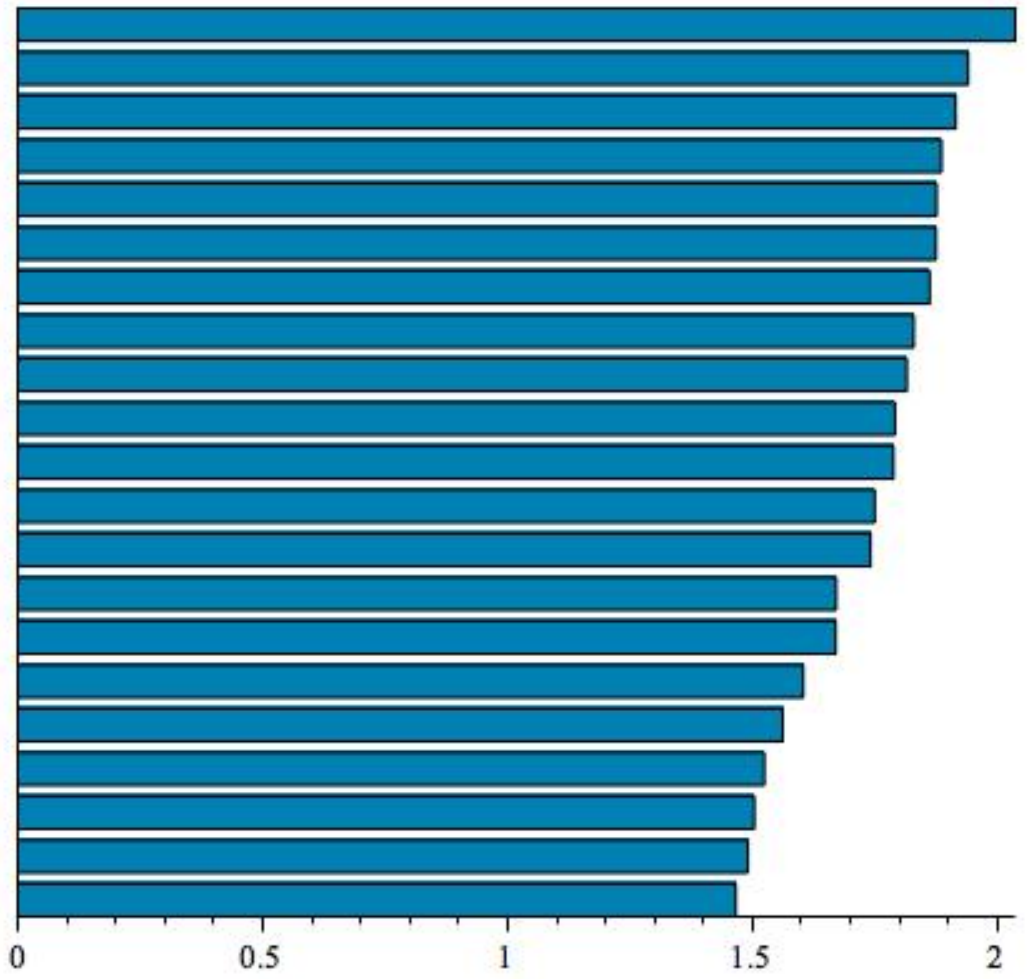

Figure 3. Corruption of syntactic parameters in a sparse distributed memory (non-normalized).

\begin{tabular}{|c|c|}
\hline Parameter & Corruption (non-normalized) \\
\hline [01] Subject-Verb & 1.50385541439 \\
\hline [02] Verb-Subject & 2.03638553143 \\
\hline [03] Verb-Object & 1.56180722713 \\
\hline [04] Object-Verb & 1.86186747789 \\
\hline [05] Subject-Verb-Object & 1.6709036088 \\
\hline [06] Subject-Object-Verb & 1.88596384645 \\
\hline [07] Verb-Subject-Object & 1.7879518199 \\
\hline [08] Verb-Object-Subject & 1.66993976116 \\
\hline [09] Object-Subject-Verb & 1.46596385241 \\
\hline [10] Object-Verb-Subject & 1.4907228899 \\
\hline [11] Adposition-Noun-Phrase & 1.52427710056 \\
\hline [12] Noun-Phrase-Adposition & 1.81512048125 \\
\hline [13] Adjective-Noun & 1.82927711248 \\
\hline [14] Noun-Adjective & 1.6037349391 \\
\hline [15] Numeral-Noun & 1.74969880581 \\
\hline [16] Noun-Numeral & 1.94036144018 \\
\hline [17] Demonstrative-Noun & 1.87596385121 \\
\hline [18] Noun-Demonstrative & 1.87463855147 \\
\hline [19] Possessor-Noun & 1.91487951279 \\
\hline$[20]$ Noun-Possessor & 1.74102410674 \\
\hline [A01] Attributive-Adjective-Agreement & 1.79102409244 \\
\hline
\end{tabular}


[17] DemonstrativeNoun

[15] NumeralNoun

[19] PossessorNoun

[13] AdjectiveNoun

[16] NounNumeral

[02] VerbSubject

[18] NounDemonstrative

[04] ObjectVerb

[06] SubjectObjectVerb

[12] NounPhraseAdposition

[AOI] AttributiveAdjectiveAgreement

[20] NounPossessor

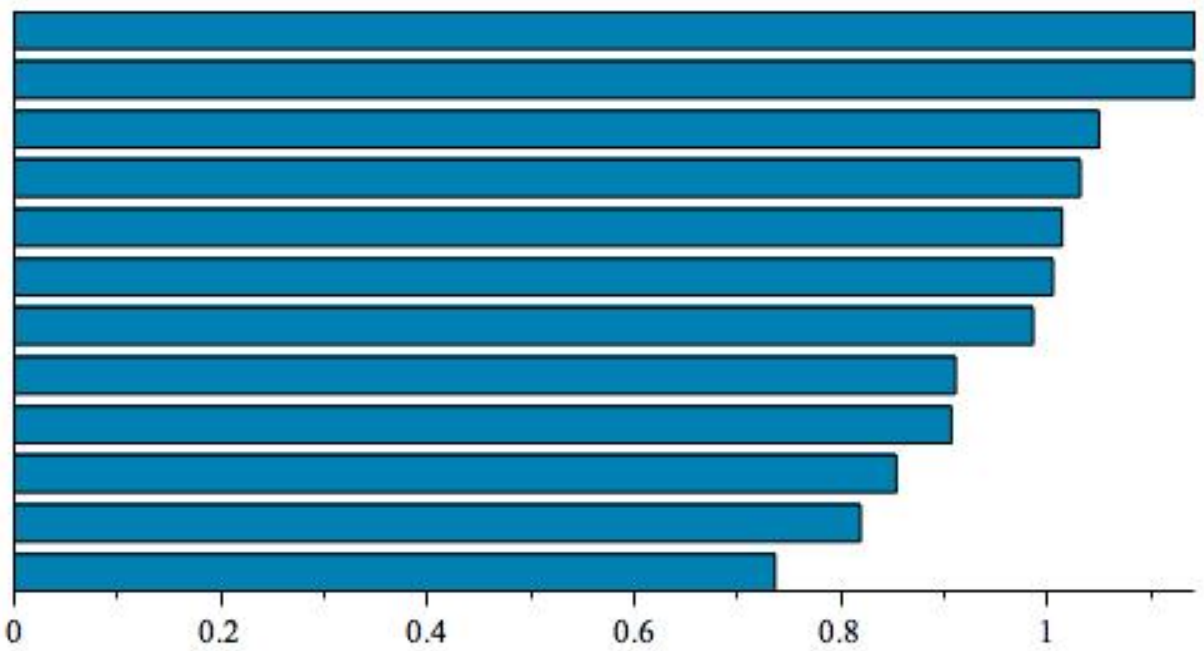

0.4

0.8

FiguRE 4. Corruption (normalized test) of some syntactic parameters.

\begin{tabular}{|c|c|}
\hline Parameter & Corruption (normalized) \\
\hline \hline$[02]$ Verb-Subject & 1.00494736842 \\
\hline$[04]$ Object-Verb & 0.910842105263 \\
\hline$[06]$ Subject-Object-Verb & 0.906736842105 \\
\hline$[12]$ Noun-Phrase-Adposition & 0.853473684211 \\
\hline$[13]$ Adjective-Noun & 1.03157894737 \\
\hline$[15]$ Numeral-Noun & 1.14094736842 \\
\hline$[16]$ Noun-Numeral & 1.01378947368 \\
\hline$[17]$ Demonstrative-Noun & 1.14157894737 \\
\hline$[18]$ Noun-Demonstrative & 0.985789473684 \\
\hline$[19]$ Possessor-Noun & 1.04957894737 \\
\hline$[20]$ Noun-Possessor & 0.736105263158 \\
\hline$[$ A01] Attributive-Adjective-Agreement & 0.818842105263 \\
\hline
\end{tabular}

\section{Further Questions And Directions}

We outline here some possible directions in which we plan to expand the present work on an approach to the study of syntactic parameters using Kanerva Networks.

6.1. Kanerva Networks and Language Families. Through our experiments of corrupting a syntactic parameter and checking whether the Kanerva Network can successfully reconstruct the original data, we have learned that the corruption of certain syntactic parameters is more fixable in the Kanerva Network. One interpretation of this result is that such parameters are dependent on the remaining ones. Indeed, for the set of syntactic parameters used in this study, we know a priori, for linguistic reasons, that there should be a certain degree of dependency between some of the parameters, for example in the case of the first group of ten parameters governing the word order relations between subject, verb, and object, with the caveat discussed in $\$ 2.2$ above on how one should interpret such relations. A more detailed study of known relations between other groups of syntactic parameters and how they correlate to measures of recoverability in a Kanerva Network would be needed in order to better understand how syntactic dependencies affect 
recoverability, and further develop Kanerva Networks as a possible approach to detect additional dependency relations between the binary variables of other syntactic parameters.

As we have seen, the scalar score we obtain from the corruption experiments indicates how tractable is a variable, or syntactic parameter, in the context of data points in its vicinity. In other words, if the scalar score is small for a certain parameter, then the parameter is derivable from other correct bits. Yet, one limitation of our result is that this scalar score is simply computed as the average of the Hamming distance between the resultant bit-vector and the original bit-vector. The derivability of a certain parameter might vary depending on the family of languages that it belongs to. For example, when a certain language feature is not robust to corruption in certain regions of the Kanerva Network, which means the parameter is not depended on other parameters, but robust to corruption in all the other regions, we will get a low scalar score.

While our present approach can provide some meaningful insight about whether a certain feature is generally retrievable by analyzing other features, it does not shed light on identifying which feature is a determining feature in a family of languages. In other words, if a feature is very tractable (low scalar score) in one family of languages, this means that feature is a sharing characteristic of the language group. If it is not very tractable, then it might indicate that the feature is a changeable one in the group. Thus, by conducting the same experiments grouped by language families, we may be able to get some information about which features are important in which language family.

It is reasonable to assume that languages belonging to the same historical-linguistic family are located near each other in the Kanerva Network. However, a more detailed study where data are broken down by different linguistic families will be needed to confirm this hypothesis.

Under the assumption that closely related languages remain near in the Kanerva Network, the average of dependencies of a given parameter over the whole space might be less informative globally, because there is no guarantee that the dependencies would hold throughout all regions of the Kanerva Network. However, this technique may help identifying specific relations between syntactic parameters that hold within specific language families, rather than universally across all languages. The existence of such relations is consistent with the topological features identified in [22] which vary across language families, so we expect to encounter similar phenomena from the Kanerva Networks viewpoint as well.

6.2. Kanerva Networks and the Language-Neuroscience Connection. One of the main open frontiers in understanding human language is relating the structure of natural languages to the neuroscience of the human brain. In an idealized vision, one could imagine a Universal Grammar being hard wired in the human brain, with syntactic parameters being set during the process of language acquisition (see [1] for an expository account). This view is often referred to as the Chomskian paradigm, because it is inspired by some of Chomsky's original proposals about Universal Grammar. There have been recent objections to the Universal Grammar model, see for instance [5]. Moreover, a serious difficulty lies in the fact that there is, at present, no compelling evidence from the neuroscience perspective that would confirm this elegant idea. Some advances in the direction of linking a Universal Grammar model of human language to neurobiological data have been obtained in recent years: for example, some studies have suggested Broca's area as a biological substrate for Universal Grammar, [20].

Moreover, recent studies like [16] have found indication of a possible link between the cross linguistic prevalence of syntactic parameters relating to word order structure and neuroscience models of how action is represented in Broca's area of the human brain. This type of results seems to cast a more positive light on the possibility of relating syntactic parameters to computational neuroscience models. 
Models of language acquisition based on neural networks have been previously developed, see for example the survey [23]. Various results, [4], [11], [14], 15], [17], have shown advantages of Kanerva's sparse distributed memories over other models of memory based on neural networks. To our knowledge, Kanerva Networks have not yet been systematically used in models of language acquisition, although the use of Kanerva Networks is considered in the work [18 on emergence of language. Thus, a possible way to extend the present model will be storing data of syntactic parameters in Kanerva Network, with locations representing (instead of different world languages) events in a language acquisition process that contain parameter-setting cues. In this way, one can try to create a model of parameter setting in language acquisition, based on sparse distributed memories as a model of human memory. We will return to this approach in future work.

\section{APPEndix: LANGUAGES}

The list of languages from the SSWL database that we considered for this study consists of: Acehnese, Afrikaans, Albanian, American Sign Language, Amharic, Ancient Greek, Arabic (Gulf), Armenian (Eastern), Armenian (Western), Bafut, Bajau (West Coast), Bambara, Bandial, Basaa, Bellinzonese, Beng, Bengali, Bole, Brazilian Portuguese, Breton, Bulgarian, Burmese, Calabrian (Northern), Catalan, Chichewa, Chol, Cypriot Greek, Czech, Dagaare, Digo, Digor Ossetic, Dutch, Eastern Armenian, English, English (Singapore), European Portuguese, Ewe, Farefari, Faroese, Finnish, French, Frisian (West Frisian), Ga, Galician, Garifuna, Georgian, German, Ghomala', Greek, Greek (Cappadocian), Greek (Homeric), Greek (Medieval), Gungbe (Porto-Novo), Gurene, Guébie, Haitian, Hanga, Hausa, Hebrew, Hindi, 'Hoan, Hungarian, Ibibio, Icelandic, Iha, Ilokano, Imbabura Quichua Indonesian, Irish, Iron Ossetic, Italian, Italian (Ancient Neapolitan), Japanese, K'iche', Karachay, Kashaya, Kayan, Khasi, KiLega, Kinande, Kiswahili, Kiyaka, Kom, Korean, Kuot, Kurdish (Sorani), Kusunda, Lango, Lani, Lao, Latin, Latin (Late), Lebanese Arabic, Lubukusu, Maasai (Kisongo), Malagasy, Mandarin, Maori, Marshallese, Masarak, Medumba, Middle Dutch, Miya, Moroccan Arabic, Muyang, Nahuatl (Central Huasteca), Naki, Nawdm, Ndut, Nepali, Northern Thai, Norwegian, Nupe, Nweh, Okinawan, Old English, Old French, Old Saxon, Oluwanga, One, Palue, Panjabi, Papuan Malay, Pashto, Pima, Polish, Q'anjob'al, Romanian, Russian, Salasaca Quichua, Samoan, San Dionisio Ocotepec Zapotec, Sandawe, Saweru, Scottish Gaelic, Senaya, Shupamem, Sicilian, Skou, Slovenian, Spanish, Swedish, Tagalog, Taiwanese Southern Min, Thai, Tigre, Titan, Tlingit, Tommo-So, Tongan Triqui Copala, Tukang Besi, Tuki (Tukombo), Tupi (Ancient), Turkish, Twi, Ukrainian, Vata, West Flemish, Wolane, Wolof, Yawa, Yiddish, Yoruba, Zulu.

\section{REFERENCES}

[1] M. Baker, The Atoms of Language, Basic Books, 2001.

[2] N. Chomsky, Lectures on Government and Binding, Dordrecht: Foris Publications, 1982.

[3] N. Chomsky, H. Lasnik, The theory of Principles and Parameters, in "Syntax: An international handbook of contemporary research", pp.506-569, de Gruyter, 1993.

[4] Ph.A. Chou, The capacity of the Kanerva associative memory, IEEE Trans. Inform. Theory, Vol. 35 (1989) N. 2, 281-298.

[5] D.L. Everett, Cultural Constraints on Grammar and Cognition in Pirahã: Another Look at the Design Features of Human Language, Current Anthropology 46 (2005) N.4, 621-646

[6] S. Franklin, Artificial Minds, MIT Press, 2001.

[7] S.B. Furber, G. Brown, J. Bose, J.M. Cumpstey, P. Marshall, J.L. Shapiro, Sparse distributed memory using rank-order neural codes, IEEE Trans. on Neural Networks, Vol. 18 (2007) N. 3, 648-659.

[8] C. Galves (Ed.) Parameter Theory and Linguistic Change, Oxford University Press, 2012.

[9] M. Haspelmath, M.S. Dryer, D. Gil, B. Comrie, The World Atlas of Language Structures, Oxford University Press, 2005. http://wals.info/ 
[10] M. Haspelmath, Parametric versus functional explanations of syntactic universals, in "The limits of syntactic variation", pp. 75-107, John Benjamins, 2008.

[11] T.A. Hely, D.J. Willshaw, G.M. Hayes, A New Approach to Kanerva's Sparse Distributed Memory, IEEE Transactions on Neural Networks, Vol. 8 (1997) N. 3, 791-794.

[12] P. Kanerva, Sparse Distributed Memory, MIT Press, 1988.

[13] P. Kanerva, Sparse Distributed Memory and Related Models, in "Associative Neural Memories: Theory and Implementation", M.H. Hassoun, Ed., pp. 50-76, Oxford University Press, 1993. http://www.rni.org/kanerva/sdmchapter-text.pdf

[14] P. Kanerva, Encoding structure in Boolean space, in "ICANN 98: Perspectives in Neural Computing (Proceedings of the 8th International Conference on Artificial Neural Networks, Skoevde, Sweden)", L. Niklasson, M. Boden, and T. Ziemke (eds.) 1, pp. 387-392, Springer 1998.

[15] J.D. Keeler, Capacity for patterns and sequences in Kanerva's SDM as compared to other associative memory models, in "Neural Information Processing Systems", Ed. D.Z. Anderson, pp. 412-421, American Institute of Physics, 1988.

[16] D. Kemmerer, The cross-linguistic prevalence of SOV and SVO word orders reflects the sequential and hierarchical representation of action in Broca's area, Language and Linguistics Compass, Vol.6 (2012) N.1, 50-66.

[17] A. Knoblauch, G. Palm, F.T. Sommer, Memory capacities for synaptic and structural plasticity, Neural Computation, Vol. 22 (2010) 289-341.

[18] B. MacWhinney, Models of the Emergence of Language, Annual Review of Psychology, 49 (1998) $199-227$.

[19] M. Marcolli, Principles and Parameters: a coding theory perspective, arXiv:1407.7169 [cs.CL]

[20] G.F. Marcus, A. Vouloumanos, I.A. Sag, Does Broca's play by the rules? Nature Neuroscience, Vol.6 (2003) N.7, 651-652.

[21] M. Mendes, A.P. Coimbra, M. Crisóstomo, AI and memory: Studies towards equipping a robot with a sparse distributed memory, IEEE Int. Conf. on Robotics and Biomimetics (ROBIO), pp. 1743-1750, Sanya, China, 2007.

[22] A. Port, I. Gheorghita, D. Guth, J.M. Clark, C. Liang, S. Dasu, M. Marcolli, Persistent Topology of Syntax, arXiv:1507.05134 [cs.CL]

[23] J. Poveda, A. Vellido, Neural network models for language acquisition: a brief survey, in "Intelligent Data Engineering and Automated Learning - IDEAL 2006", Lecture Notes in Computer Science, Vol.4224, Springer, 2006, pp. 1346-1357.

[24] R. Prager, F. Fallside, The modified Kanerva model for automatic speech recognition, Computer Speech and Language, Vol. 3 (1989) 61-81.

[25] D. Rogers, Predicting Weather Using a Genetic Memory: a Combination of Kanerva's Sparse Distributed Memory with Holland's Genetic Algorithms, in "Connectionist Models. Proceedings of the 1990 Summer School", Ed. D.S. Touretzky, pp. 455-464, Morgan Kaufmann, 1990.

[26] K. Siva, J. Tao, M. Marcolli, Spin Glass Models of Syntax and Language Evolution, arXiv:1508.00504 [cs.CL]

[27] A. Taylor, The change from SOV to SVO in Ancient Greek, Language Variation and Change, Vol.6 (1994) $1-37$.

[28] R. Tomlin, Basic Word Order: Functional Principles, Croom Helm, 1986.

[29] Syntactic Structures of World Languages (SSWL Database) http://sswl.railsplayground.net/ recently migrated to TerraLing

[30] TerraLing Database http://www.terraling.com/

Division of Physics, Mathematics, and Astronomy, California Institute of Technology, 1200

E. California Blvd, Pasadena, CA 91125, USA

E-mail address: jpark3@caltech.edu

E-mail address: ronnel@caltech.edu

E-mail address: azhao@dmail.caltech.edu

E-mail address: axlemn@gmail.com

E-mail address: kyuh@caltech.edu

E-mail address: vibhor@caltech.edu

E-mail address: matilde@caltech.edu 